\title{
Study of Micro Cantilever Beam Sizes Effect on Pull-In Voltage
}

\author{
Meng-Ju Lin', Wei Jing Chiu ${ }^{1}$ \\ ${ }^{1}$ Department of Mechanical and Computer Aided Engineering, Feng Chia University \\ No. 100 WenHua Rd., Taichung, Taiwan \\ mengjlin@fcu.edu.tw; skylove5237@mail.fcu.edu.tw
}

\begin{abstract}
Due to the importance of electrostatically actuated method used in MEMS, pull-in phenomena of devices needs more investigation. Effects of cantilever beam sizes on pull-in voltages are analysed in this work. Numerical simulation by using finite element software COMSOL Multiphysics is provided to analyze the pull-in phenomena. The results show that pull-in voltages will increase a little nonlinearly as gap between cantilever beam and ground electrode increasing. Initially, as the gap increasing, pull-in voltage increases with larger rate. But the increasing rate will decrease as gap increasing. If the gap is larger than $2 \mu \mathrm{m}$, the increasing rate will become almost constant. That is, the pull-in voltage will increase linearly like as gap increasing larger than $2 \mu \mathrm{m}$. And the pull-in voltage would increase linearly like for beam thickness increasing. Gap and beam thickness have almost the same effect on pull-in voltage. However, the beam width has the tiniest effect on pull-in voltage comparing with the other parameters. It is found that the pull-in voltages are almost the same as beam width increasing. And pull-in voltage decreases nonlinearly as beam length increasing. For the longer beam length, the pull-in voltage will reaches a limit values as beam length increasing. The beam length has the most significant effect on pullin voltage.
\end{abstract}

Keywords: pull-in phenomenon, electrostatically actuated, COMSOL Multiphysics, cantilever beam

\section{Introduction}

Micro-electromechanical systems (MEMS) is important for its reducing size, weight and power consumption of devices and being actuated by miniaturized by components and system. Therefore, it is widely used and has massive products. The MEMS devices have many commercial applications, such as micro-switches, pressure sensor, micro mirrors, mass sensors, micro energy harvesters, micro resonators, and biosensors. For actuators, there are some driving methods used. The devices can be actuated using thermal [1], piezoelectric [2] or electrostatic methods [3-4]. Where the electrostatic actuation is mostly used for its simplicity, small space and negligible mass as well as accessibility to high frequency actuation [5-7], where the electrostatically actuated method is often utilized in MEMS. However, for electrostatically actuated MEMS, the terrible pullin phenomena will happen in MEMS as devices being actuated. The pull-in phenomena are an unstable dynamic behaviour. As applying and increasing voltages on actuators, the electrostatic force will increase nonlinearly as gap between electrode reducing. However, the elastic force increases linearly. As the gap reaching a critical value, the electrostatic force is larger than elastic force. The two electrodes will close to each other very fast and finally contact. The phenomena are called pullin phenomena. The pull-in phenomena will destroy the devices or diminish performance of actuators. Therefore, how to avoid pull-in is very important. Although this phenomenon is the main principle of the performance of micro-switches [8], it is impediment to appropriate performance of devices such as resonators, pressure gauges and so on. [9]. Therefore, investigating pull-in is significantly prior to design and fabrication of MEMS. Moreover, nonlinearity is an important topic of MEMS dynamics [10]. The size effect on pull-in phenomena is important in MEMs. A study by the experimental and theoretical comparisons of pull-in deflection of the beam [11] is investigated. The Taguchi method is also used to optimize the design of the cantilever beam [12]. The statistical ANOVA method was used to simulate the deformation caused by the electrostatic force. It was found that the main factors affecting the displacement of the cantilever were the size of the cantilever beam. The nonlinear differential control equations with Hamilton's principle are derived [13]. The results show that the smaller the size of the cantilever beam, the much easier of pull-in phenomena happening. The nonlinear equation of motion is derived and analysed [14]. The pull-in instability and nonlinear dynamics of the microcantilever are discussed. It is concluded that the cantilever beam sizes have significant effect on pull-in voltage and dynamic response. Due to the importance of electrostatically actuated method used in MEMS, pull-in phenomena of devices needs more investigation. 
Effects of cantilever beam sizes on pull-in voltages are analysed in this work. Numerical simulation by using finite element software COMSOL Multiphysics is provided to analyze the pull-in phenomena.

\section{Numerical model}

A typical cantilever beam is shown in Figure 1 (a). The length, the width and the thickness of the cantilever beam are $L, w$ and $t$, respectively. The initial gap between the cantilever beam and ground electrode is $g$. The upper cantilever beam is the actuating electrode for applying voltage. The substrate beneath the cantilever beam is used as ground electrode. Using a finite element method software COMSOL Multiphysics as simulation tool, the numerical model is shown in Figure 1 (b). The numerical model is automeshed as shown in Figure (c). After applying voltage, the cantilever beam will be deformed. Its deflection will be expressed in different collars as Figure (d). The material of the structure is single crystalline silicon. Its material properties are expressed in Table 1. As Figure 1 (d) shown, the maximum deflection happens at free end of cantilever beam. When the applying voltages increase, the deflections at free end also increase. If the deflection equals to the gap size, it means the pull-in phenomena happen. The applying voltage is called pull-in voltage. The effect of cantilever beam sizes on pull-in voltages can be investigated by the numerical model.

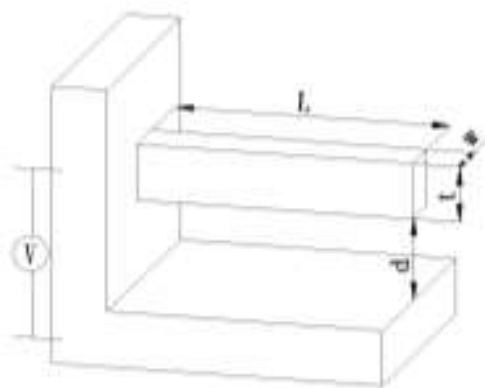

(a)

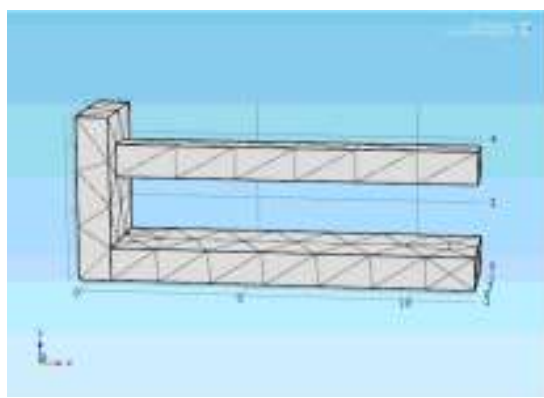

(c)

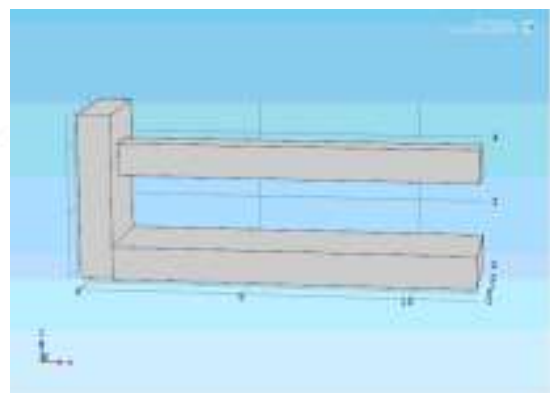

(b)

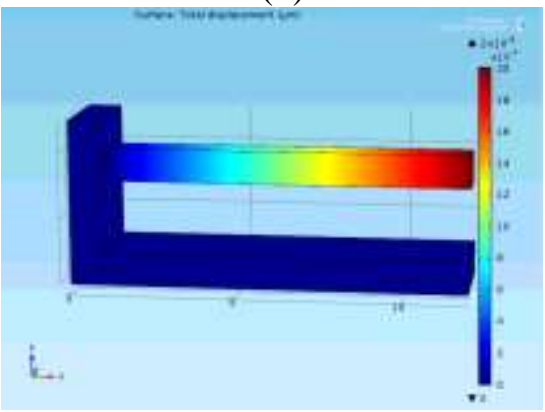

(d)

Fig. 1: (a) Schematic of the cantilever beam. (b) Numerical model. (c) Automeshed numerical model. (d) Deflection of cantilever beam.

Table 1: Material properties of single crystalline silicon.

\begin{tabular}{|c|c|c|c|}
\hline Property & Name & Value & Unit \\
\hline Relative permittivity & $\varepsilon_{\mathrm{r}}$ & 11.7 & 1 \\
\hline Density & $\rho$ & 2330 & $\mathrm{~kg} / \mathrm{m}^{3}$ \\
\hline Young's modulus & $\mathrm{E}$ & $170 \times 10^{9}$ & $\mathrm{~Pa}$ \\
\hline Poisson's ratio & $v$ & 0.28 & 1 \\
\hline
\end{tabular}




\section{Results and discussions}

Effect of cantilever beam sizes on pull-in voltages is shown in Figure 2 (a) to (d). Figure 2 (a) shows the effect of gap on pull-in voltage. Under the same size of the cantilever beam width, thickness, and length, it is shown that the greater the gap, the larger the pull-in voltage. As shown in this figure, the pull-in voltage increases a little nonlinearly as gap increasing. Initially, as the gap increasing, pull-in voltage increases with larger rate. But the increasing rate will decrease as gap increasing. If the gap is larger than $2 \mu \mathrm{m}$, the increasing rate will become almost constant. That is, the pull-in voltage will increase linearly like as gap increasing larger than $2 \mu \mathrm{m}$. And the pull-in voltage would increase linearly like for beam thickness increasing as shown in Figure 2 (b). Gap and beam thickness have almost the same effect on pull-in voltage. However, the beam width has the tiniest effect on pull-in voltage comparing with the other parameters. As shown in Figure 2 (c), it is found that the pull-in voltages are almost the same as beam width increasing. The effect of beam width on pull-in voltage is not so significant comparing with gap and beam thickness. And pull-in voltage decreases nonlinearly as beam length increasing as shown in Figure 2 (d). The pull-in voltage decreases quickly when beam length is short. However, as beam length larger than $120 \mu \mathrm{m}$, the pull-in voltages decreasing rate become smaller and pull-in voltage would approach a limit value. Therefore, the beam length has the most significant effect on pull-in voltage.

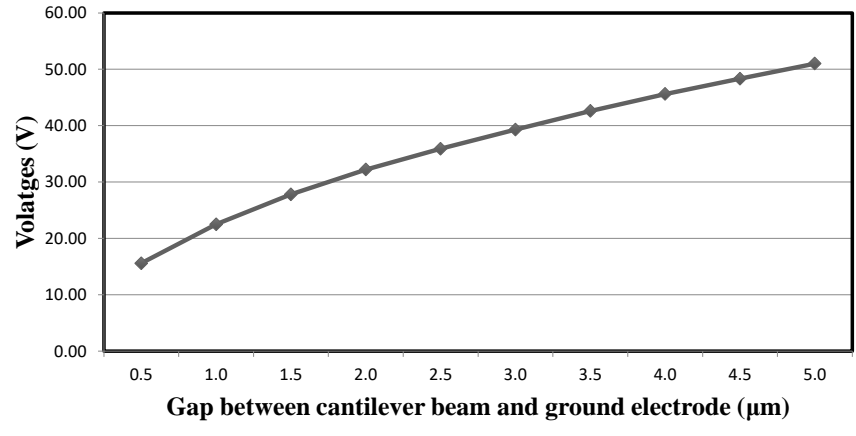

(a)

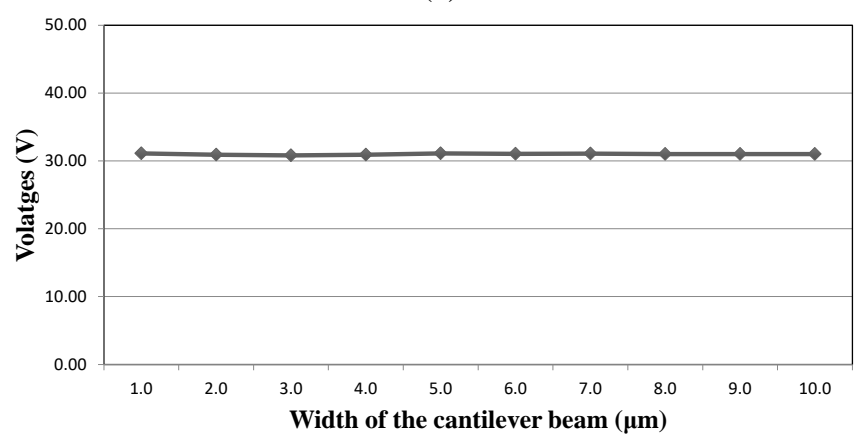

(c)

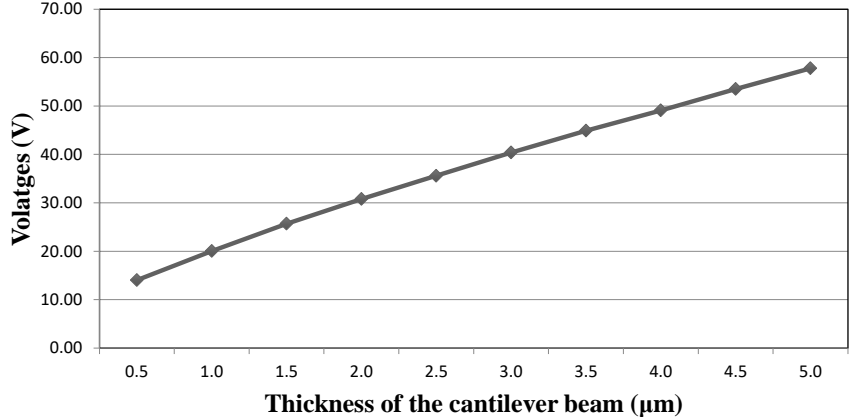

(b)

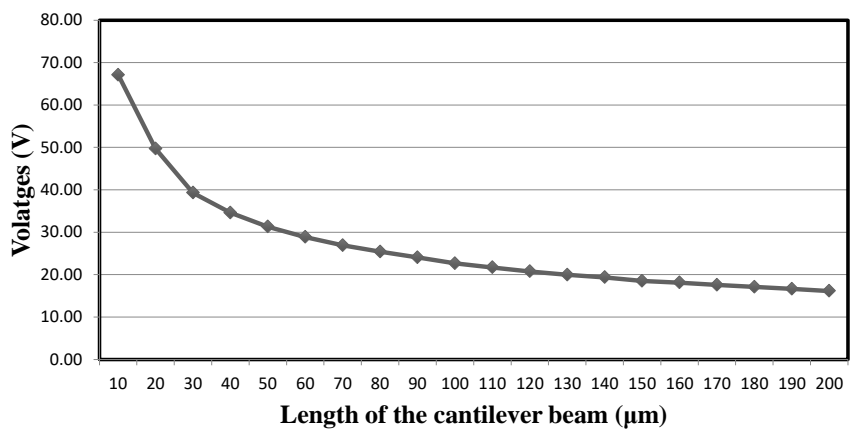

(d)

Fig. 2: (a) Effect of gap on pull-in voltage. ( $\mathrm{L}=50 \mu \mathrm{m}, \mathrm{w}=4 \mu \mathrm{m}, \mathrm{t}=2 \mu \mathrm{m})$ (b) Effect of beam thickness on pull-in voltage. $(\mathrm{L}=50$ $\mu \mathrm{m}, \mathrm{w}=4 \mu \mathrm{m}, \mathrm{d}=2 \mu \mathrm{m})(\mathrm{c})$ Effect of beam width on pull-in voltage. $(\mathrm{L}=50 \mu \mathrm{m}, \mathrm{t}=2 \mu \mathrm{m}, \mathrm{d}=2 \mu \mathrm{m})(\mathrm{d})$ Effect of beam length on pull-in voltage. $(\mathrm{w}=4 \mu \mathrm{m}, \mathrm{t}=2 \mu \mathrm{m}, \mathrm{d}=2 \mu \mathrm{m})$.

\section{Conclusion}

Effects of cantilever beam sizes on pull-in voltages are investigated. The width of the cantilever beam has the least effect on the pull-in voltage. Different beam width would have almost the same pull-in voltage. The length has significant influence on the pull-in voltage. The pull-in voltages will nonlinearly decrease as beam length increasing. Pull-in voltage will increase as beam thickness and gap increasing. And the increasing rates for gap and beam thickness are similar. Their relations show linearly like increasing. 


\section{References}

[1] S. Muratet, S. Lavu, J-Y. Fourniols, G. Bell, M. P. Y. Desmulliez, "Reliability modelling and analysis of thermal MEMS," J. Phys. Conf. Ser., 2006.

[2] S. Azizi, M-R. Ghazavi, S. E. Khadem, J. Yang, G. Rezazadeh, "Stability analysis of a parametrically excited functionally graded piezoelectric, MEM system," Curr. Appl. Phys., vol. 12, no. 2, pp. 456-466, 2012.

[3] M. Younis, E.M., Abdel-Rahman, A. Nayfeh, "A reduced-order model for electrically actuated microbeam-based MEMS," J. Micromech. Syst., vol. 12, no. 5, pp. 672-680, 2003.

[4] G. Rezazadeh, A. Tahmasebi, M. Zubstov, "Application of piezoelectric layers in electrostatic MEM actuators: controlling of pull-in voltage," Microsyst. Technol., vol. 12, no. 12, pp. 1163-1170, 2006.

[5] S.W. Lee, E.E. Campbell, "Nanoelectromechanical devices with carbon nanotubes," Curr. Appl. Phys., vol. 13, no. 8, pp. 1844-1859, 2013.

[6] E. M. Miandoab, H. N. Pishkenari, A. Yousefi-Koma, F. Tajaddodianfar, "Chaos prediction in MEMS-NEMS resonators," Int. J. Eng. Sci., vol. 82, pp. 74-83, 2014.

[7] H. Dai, L. Wang, "Surface effect on the pull-in instability of cantilevered nano-switches based on a full nonlinear model," Phys. E Low-Dimens. Syst. Nanostruct., 2015.

[8] E. Yazdanpanahi, A. Noghrehabadi, M. Ghalambaz, "Balance dielectric layer for micro electrostatic switches in the presence of capillary effect," Int. J. Mech. Sci., vol. 74, pp. 83-90, 2013.

[9] X. Guo, Y.-B. Yi, S. Pourkamali, "A finite element analysis of thermoelastic damping in vented MEMS beam resonators," Int. J. Mech. Sci., vol. 74, pp. 73-82, 2013.

[10] Ehsan Maani Miandoab, Hossein Nejat Pishkenari, Ali Meghdari, Mohammad Fathi, "A general closed-form solution for the static pull-in voltages of electrostatically actuated MEMS/NEMS," Physica E: Low-dimensional Systems and Nanostructures, vol. 90, pp. 7-12, 2017.

[11] Lior Medina, Rivka Gilat, Bojan Ilic and Slava Krylov, "Experimental investigation of the snap-through buckling of electrostatically actuated initially curved pre-stressed micro beams," Sensors and Actuators A: Physical, vol. 220, pp. 323-332, 2014.

[12] Deepak G.Khushalani, Vaibhav R.Dubey, Jayu P.Kalambe, Rajesh S.Pande and Rajendra M.Patrikar, "Design optimization \& fabrication of micro cantilever for switching application," Sensors and Actuators A: Physical, vol. 225, pp. 1-7, 2015.

[13] Yue Xiao, Binglei Wang and Shenjie Zhou, "Pull-in voltage analysis of electrostatically actuated MEMS with piezoelectric layers: A size-dependent model," Mechanics Research Communications, vol. 66, pp. 7-14, 2015.

[14] H. L. Dai and L. Wang, "Size-dependent pull-in voltage and nonlinear dynamics of electrically actuated microcantilever-based MEMS: A full nonlinear analysis," Communications in Nonlinear Science and Numerical Simulation, vol. 46, pp. 116-125, 2017. 